\title{
Correction to: Whole genome sequences of two Trichophyton indotineae clinical isolates from India emerging as threats during therapeutic treatment of dermatophytosis
}

\author{
Pawan Kumar $^{1,2} \cdot$ Shukla Das $^{3} \cdot$ Richa Tigga $^{3,5} \cdot$ Rajesh Pandey $^{1} \cdot$ S. N. Bhattacharya ${ }^{4} \cdot$ Bhupesh Taneja $^{1,2} \mathbb{D}$ \\ Published online: 30 October 2021 \\ ○ King Abdulaziz City for Science and Technology 2021
}

Correction to: 3 Biotech (2021) 11:402

https: //doi.org/10.1007/s13205-021-02950-1

In the Original Publication, the Table 2 has some error. The correct Table 2 is as follows:

The original article can be found online at https: //doi.org/10.1007/ s13205-021-02950-1.

\section{Bhupesh Taneja}

btaneja@igib.res.in

1 CSIR-Institute of Genomics and Integrative Biology, New Delhi 110025, India

2 Academy of Scientific and Innovative Research (AcSIR), Ghaziabad 201002, India

3 Department of Microbiology, UCMS and GTB Hospital, Dilshad Garden, Delhi 110095, India

4 Department of Dermatology, UCMS and GTB Hospital, Dilshad Garden, Delhi 110095, India

5 Present Address: Department of Microbiology, All India Institute of Medical Sciences, Raipur, India 
Table 2 Standard checklist of the Minimal Information about any (X) Sequence (MIxS) for Trichophyton indotineae UCMS-IGIB-CI12 and Trichophyton indotineae UCMS-IGIB-CI14

Trichophyton indotineae TiCI12

Classification

Collection Date

Geographical Location (latitude and longitude)

Geographical Location (Country)

Environment (biome)

Environment (feature)

Environmental(Material)

MIxS data Investigation

Submitted to insdc

Investigation type

Project name

BioProject

BioSample

Depth

Ploidy

Isolation and Growth Condition

Sequencing method

Assembly

Finishing Strategy
Kingdom: Fungi

Division: Ascomycota

Class: Eurotiomycetes

Order: Onygenales

Family: Arthrodermataceae

Genus: Trichophyton

Species: T. indotineae

Strain: UCMS-IGIB CI12

\section{4-09-25}

$28.7041 \mathrm{~N} 77.1025 \mathrm{E}$

Delhi, India

Homo sapiens

Skin

Lesion

\section{JAATJQ000000000.1 (GenBank)}

Fungi

Whole genome sequence of Trichophyton indotineae CI12 from India

PRJNA604098

SAMN13951834

143.7x

Diploid

SDA with chloramphenicol, gentamicin and cycloheximide

Illumina HiSeq

SPAdes v. 3.13 assembler

Draft, over 143.7-fold genome coverage, 824 contigs
Trichophyton indotineae TiCI14

Kingdom: Fungi

Division: Ascomycota

Class: Eurotiomycetes

Order: Onygenales

Family: Arthrodermataceae

Genus: Trichophyton

Species: T. indotineae

Strain: UCMS-IGIB CI14

2014-09-30

$28.7041 \mathrm{~N} 77.1025 \mathrm{E}$

Delhi, India

Homo sapiens

Skin

Lesion

JAAQVJ000000000.1 (GenBank)

Fungi

Whole genome sequence of Trichophyton indotineae CI14 from India

PRJNA604102

SAMN13951939

$136.3 \mathrm{x}$

Diploid

SDA with chloramphenicol, gentamicin and cycloheximide

Illumina HiSeq

SPAdes v. 3.13 assembler

Draft, over 136.3-fold genome coverage, 904 contigs 